\title{
Psychometric Properties of the Greek Version of the Obsessive-Compulsive Inventory-Revised in a Non-Clinical Young Adult Sample
}

\author{
Gregoris Simos$^{1 *}$, Olga Zikopoulou${ }^{1}$, Anna Nisyraiou1, Konstantinos Zafiropoulos² \\ ${ }^{1}$ Department of Educational and Social Policy, University of Macedonia, Thessaloniki, Greece \\ ${ }^{2}$ Department of International and European Studies, University of Macedonia, Thessaloniki, Greece \\ Email: ^gsimos@uom.edu.gr
}

How to cite this paper: Simos, G., Zikopoulou, O., Nisyraiou, A., \& Zafiropoulos, K. (2019). Psychometric Properties of the Greek Version of the Obsessive-Compulsive Inventory-Revised in a Non-Clinical Young Adult Sample. Psychology, 10, 2247-2265. https://doi.org/10.4236/psych.2019.1016142

Received: October 29, 2019

Accepted: December 27, 2019

Published: December 30, 2019

Copyright $\odot 2019$ by author(s) and Scientific Research Publishing Inc. This work is licensed under the Creative Commons Attribution International License (CC BY 4.0).

http://creativecommons.org/licenses/by/4.0/

\begin{abstract}
Obsessive-Compulsive Inventory-Revised (OCI-R) is a self-report questionnaire that assesses the distress caused by a variety of OCD symptoms; OCI-R comprises of 18 items that are equally divided into six subscales: Checking, Washing, Obsessing, Neutralization, Ordering, and Hoarding. Research so far has shown that OCI-R is a psychometrically sound measure with excellent validity and reliability. It is also appropriate for clinical and non-clinical populations and has been translated into several languages and has been validated in diverse cultural contexts. The aim of the present study was to validate OCI-R in the Greek language and to examine its psychometric properties in a Greek non-clinical sample. Three hundred and seventy one university students participated in this study. Measures used were OCI-R, the Padua Inventory (PI), the Obsessive Beliefs Questionnaire-44 (OBQ-44), and the Depression Anxiety Stress Scale (DASS-21). Consequent analysis of collected data examined the factor structure of OCI-R and tested its convergent and divergent validity. The confirmatory factor analysis showed good fit for the model and replicated the results of other studies, meeting thus the requirements for the six-factor solution of the original scale. The Greek version of OCI-R maintained the good psychometric properties of the original English one. Internal consistency was excellent for the overall scale. Convergent and divergent validity was good and also confirmed previous similar findings.
\end{abstract}

\section{Keywords}

OCI-R, Greek, Psychometric Properties, Validation, OBQ-44 


\section{Introduction}

Obsessive-Compulsive Disorder (OCD) according to the American Psychiatric Association's Diagnostic and Statistical Manual of Mental Disorders, Fifth Edition (DSM-5) is characterised by various obsessions and compulsions (APA, 2013). OCD shows a rather great heterogeneity with diverse symptom contents (Bloch, Landeros-Weisenberger, Rosario, Pittenger \& Leckman, 2008). Clinicians and researchers suggest that OCD symptoms may be classified in distinctive subtypes, such as contamination and washing/cleaning, harm obsessions and checking rituals, obsessions without overt compulsions, or hoarding subtypes (McKay, Abramowitz, Calamari, Kyrios, Radomsky et al., 2004). However, there is still no consensus whether OCD symptom contents should be partitioned into subtypes or symptom dimensions and how many they are (Olatunji, Williams, Haslam, Abramowitz, \& Tolin, 2008; Gönner, Ecker, \& Leonhart, 2009).

Moreover, it has been found that OCD has high comorbidity with other disorders, such as anxiety disorders and depression, and thus differential diagnosis is often a quite challenging task (Clark, 2004). In addition, there is a tendency of OCD patients to conceal their symptoms, not seek a timely professional help; duration of untreated OCD remains one of the highest among serious mental disorder (Altamura, Buoli, Albano, \& Dell'Osso, 2010). Most adults with OCD get effective treatment on average ten years after the onset of the first symptoms (García-Soriano, Rufer, Delsignore, \& Weidt, 2014). Additionally, Wahl et al. (Wahl, Kordon, Kuelz, Voderholzer, Hohagen, \& Zurowski, 2010) found that in outpatient clinics over $70 \%$ of OCD patients remain unrecognised and thus untreated by consultants. OCD symptoms seem to be important factors that underlie the need for early detection and treatment intervention (Belloch, del Valle, Morillo, Carrió, \& Cabedo, 2008).

A variety of questionnaires and scales have been designed to facilitate an early and accurate assessment of OCD symptoms-e.g. the Maudsley Obsessional Compulsive Inventory-MOCI (Hodgson \& Rachman, 1977), the Padua Inventory-PI (Sanavio, 1988), the Yale-Brown Obsessive-Compulsive Scale-YBOCS (Goodman, Price, Rasmussen, Mazure, Fleischmann et al., 1989), the Padua Inventory-Washington State University Revision-PI-WSUR (Burns, Keortge, Formea, \& Sternberg, 1996), the Obsessive-Compulsive Inventory-OCI (OCI, Foa, Kozak, Salkovskis, Coles, \& Amir, 1998), the Vancouver Obsessional Compulsive Inventory-VOCI (Thordarson, Radomsky, Rachman, Shafran, Sawchuk et al., 2004), the Clark-Beck Obsessive-Compulsive Inventory-C-BOCI (Clark, Antony, Beck, Swinson, \& Steer 2005), the Dimensional Obsessive-Compulsive Scale-DOCS (Abramowitz, Deacon, Olatunji, Wheaton, Berman et al., 2010).

The OCI is a self-report questionnaire that assesses on a 5-point (0-4) Likert scale OCD symptom frequency and associated distress, and it consists of 42 items that are grouped under 7 subscales: Washing, Checking, Doubting, Ordering, Obsessing, Hoarding, and Mental Neutralizing (Foa et al., 1998). There is evidence that $\mathrm{OCI}$ is a sound psychometric tool with high validity and reliability 
for both clinical and non-clinical population (Foa et al., 1998; Simonds, Thorpe, \& Elliott, 2000). However, OCI presented some disadvantages concerning its length and the time needed for one to respond to it.

Foa et al. (Foa, Huppert, Leiberg, Langner, Kichic et al., 2002) developed a shorter version containing 18items, the Obsessive-Compulsive Inventory-Revised (OCI-R). The OCI-R consists of six factors and its factor structure has been replicated in several studies. OCI-R has demonstrated good psychometric properties in clinical samples (Abramowitz, Tolin, \& Diefenbach, 2005; Abramowitz \& Deacon, 2006) and non-clinical samples (Gönner, Ecker, \& Leonhart, 2009; Hajcak, Huppert, Simons, \& Foa, 2004; Smári, Ólason, EyPórsdóttir, \& Frölunde, 2007). Consequently it is not surprising that OCI-R is one of the most commonly used self-report measures of OCD symptoms. Research on OCI-R indicated good sensitivity and specificity for cut-off scores pointing out its diagnostic sensitivity. The OCI-R subscales are valid measures of six symptom subtypes of OCD; subscales are reliable and valid measures that adequately differentiate between DSM-5 diagnostic groups. OCI-R is, therefore, psychometrically sound and, most importantly, a brief tool that is appropriate for clinical and non-clinical populations, and for clinical and research purposes (Abramowitz, et al., 2005; Abramowitz \& Deacon, 2006; Gönner et al., 2009; Hajcak et al., 2004; Smári et al., 2007).

In their recent review on evidence-based assessment of Obsessive-Compulsive Disorder, Rapp et al. (Rapp, Bergman, Piacentini, \& McGuire, 2016) conclude that the OCI- $\mathrm{R}$ is a brief self-report measure that possesses reliability, validity, and diagnostic sensitivity (with a total score of 21 corresponding to an OCD diagnosis), shows good to fair convergence with clinician-rated measures of OCD severity, but fair to poor discriminant validity from depression, anxiety, and worry.

Considerable evidence exists to suggest that the need for multilanguage versions of psychological tools is growing (Hambleton, Merenda, \& Spielberger, 2004). Cross-cultural research grows and international exchanges become more common, therefore the demand for culturally appropriate tools becomes undisputed as it establishes fairness in assessment and is time and cost-effective (Ercikan, 2002; Hambleton et al., 2004). An increasing number of scales have been translated into various languages and validated in other cultural contexts (Sousa \& Rojjanasrirat, 2011). This adaptation is important, because replication of research across different cultures requires the use of measures that have been standardized and validated in these cultures (Arafat, Chowdhury, Qusar, \& Hafez, 2016).

In that context, OCI-R has been the subject of researchers from different cultural contexts and languages. Studies conducted with both clinical and non-clinical samples provided evidence that OCI-R had good psychometric properties (Fullana, Tortella-Feliu, Caseras, Andión, Torrubis et al., 2005; Huppert, Walther, Hajcak, Yadin, Foa et al., 2007; Gönner, Leonhard, \& Ecker, 2008; Sica, Ghisi, Altoè, Chiri, Franceschini et al., 2009; Woo, Kwon, Lim, \& Shin, 
2010; Souza, Foa, Meyer, Niederauer \& Cordioli, 2011; Belloch, Roncero, García-Soriano, Carrió, Cabedo et al., 2013).

More specifically, Fullana et al. (2005) in their study of psychometric properties of the Spanish version of OCI-R in a non-clinical sample found similar fit indices in their Confirmatory Factor Analysis for the 6-factor model as Foa et al. (2002) and Hajcak et al. (2004). They also found good convergent validity and adequate divergent validity, large to moderate internal consistency, and good test-retest reliability. Huppert et al. (2007) also studied a clinical sample and concluded that the OCI-R was a psychometrically sound instrument appropriate for clinical and non-clinical populations, and for clinical and research purposes.

Gönner and colleagues (2008) conducted a study for the validation of the German version of OCI-R in a sample of people with OCD, Anxiety disorders, and Depressive disorders. They found good psychometric properties and excellent fit in their factor analysis for the OCD sample, and good properties in the anxiety and depressive disorders samples. They also found good internal consistency and good construct validity (convergent/divergent) with a slight overlap in divergent validity with other tests that are not meant to measure obsessions or compulsions such as the Beck Depression Inventory (Beck, Rush, Shaw, \& Emery, 1979), the Beck Anxiety Inventory (Beck et al., 1979), and the Penn State Worry Questionnaire (Meyer, Miller, Metzger, \& Borkovec, 1990). Overall, they concluded that OCI-R is a psychometrically sound and valid measure for use in a German population.

Sica et al. (2009) in their study with both clinical and non-clinical Italian samples confirmed the six-factor model in the Italian version of OCI-R, and also that the 6-factor structure of OC symptoms remains invariant across different cultures. OCI-R had good internal consistency and excellent temporal stability in their community sample and excellent internal consistency in their OCD sample. OCI-R showed adequate convergent validity and adequate divergent validity-correlations among OCI-R and measures of anxiety, depression and worry were small to medium in size $(.45, .41$ and .43 respectively).

The Woo et al. (2010) study confirms similar findings for the Korean version of OCI-R. In their research, they examined the psychometric properties and the effects of gender and cultural differences in both a clinical and a non-clinical sample. The results of their factor analysis indicated good fit in the six-factor model. They also found good OCI-R convergent and divergent validity, internal consistency and test-retest reliability.

Souza et al. (2011), in their study in Brazil, examined the psychometric properties of the Portuguese version of the OCI-R. For this purpose, they recruited both a clinical sample including three groups (OCD, Panic Disorder, Social Phobia), and a non-clinical sample. Subjects from the OCD sample received a 12-week Cognitive Behavioural Group Therapy (CBGT) and also completed OCI-R before and after treatment. In each sample, the overall OCI-R and subscale scores showed moderate to good internal consistency. Nevertheless, although the OCI-R Obsessing subscale had a good internal consistency in the 
non-clinical sample (Alpha $=.61$ ), Alphas in the three clinical samples were lower than .50. OCI-R total score showed good convergent and divergent validity; Spearman's rs were .40 and .47 for the correlation of OCI-R with the Y-BOCS Obsessions and Compulsions subscales respectively, and .38 and .35 for the correlation of OCI-R with BAI and BDI respectively. Finally, the OCI-R was also sensitive in assessing the effects of CBGT with OCD patients, and thus it might be helpful for measuring treatment outcomes.

Belloch et al. (2013) conducted a similar study for the Spanish version, testing OCI-R's reliability, validity, diagnostic accuracy and sensitivity to treatment effects. Participants were patients with OCD, Anxiety disorder, and a non-clinical sample. Internal consistency was excellent in the OCD sample, but less satisfactory in the two non-OCD groups. Concerning convergent and divergent validity, authors found good convergent validity for the OCI-R total score and subscales, but somewhat poor divergent validity that is significant and weak-to-moderate associations with the Beck Depression Inventory and the Penn State Worry Questionnaire. Finally, they also found excellent discriminatory power for OCI-R and excellent sensitivity to treatment.

Further studies with exclusively non-clinical samples have provided evidence that OCI-R retains good psychometric properties as a tool outside a clinical context. Zermatten et al. (Zermatten, Van der Linden, Jermann, \& Ceschi, 2006) in their research for the validation of the French version in a non-clinical sample found excellent internal consistency, while the inter-item correlations indicated medium to large effects. Interestingly, and probably due to rather cultural differences or unidentified contextual variables, mean OCI-R scores were generally lower in the French population than those obtained in non-clinical individuals by Foa et al. (2002) or Hajcak et al. (2004). Such finding further underlies the importance of the validation of clinical tools in the language and culture that they are used.

Peng et al. (Peng, Yang, Miao, Jing, \& Chan, 2011) aimed to validate a Chinese version of the OCI-R in both non-clinical and clinical samples. Their results suggested that the Chinese version of the OCI-R has a good fit to the model suggested by the original study; confirmatory factor analysis confirmed the six-factor structure of the OCI-R, namely Washing, Checking, Ordering, Obsessing, Neutralizing, and Hoarding. More importantly, their study also confirmed that the structure of OCD symptoms is similar across different cultures.

Other relevant studies have been conducted all over the world, including validation of the OCI-R in student samples-e.g in Icelanders (Smári et al., 2007) or in Persian (Farshi) language (Ghassemzadeh, Shams, Abedi, Karamghadiri, Ebrahimkhani et al. 2011), and clinical samples-e.g. in African-Americans, (Williams, Davis, Thibodeau, \& Bach, 2013), or both clinical and non-clinical samples-e.g. in Turkish (Aydin, Boysan, Kalafat, Selvi, Beşıroğlu et al, 2014). These studies have unanimously agreed that OCI-R is a useful screening tool, and also able to discriminate between patients diagnosed with OCD, patients with other anxiety disorders, and healthy individuals. 
Finally, recent work on a Greek version of OCI-R (Angelakis, Panagioti, \& Austin, 2017) also confirmed that OCI-R is a sound psychometric tool with good psychometric properties in a non-clinical community sample.

To the best of our knowledge, there are not many studies with regard to the presence of OCD in youth and young adult Greek population. Of relative interest are two epidemiological studies on prevalence and sociodemographic associations of common mental disorders (including OCD) in a nationally representative sample of the general population (Skapinakis, Bellos, Koupidis, Grammatikopoulos, Theodorakis et al., 2013), and on the epidemiology and comorbidity of obsessive-compulsive disorder in late adolescence (Politis, Magklara, Petrikis, Michalis, Simos et al., 2017).

Additionally, and concerning Greece, it seems that there is a comparative lack of validated measures for OCD, both in research and clinical practice. The only exception is the already mentioned Angelakis et al. (2017) study. This research was published during the time we had already collected our data, and had already presented part of our results on the Greek version of OCI-R (Simos \& Alexandri, 2014). Due to differences in our measures and sample, exclusively university students and thus young in age in our sample vs a 18 - 83 years old sample in the Angelakis et al. study, we decided to continue, aiming also to add more data and findings to the research on the Greek version of OCI-R.

The aim of the present study was to validate OCI-R in Greek language and to examine its psychometric properties in a Greek non-clinical young adult population. We aimed at examining 1) the factor structure of OCI-R and hopefully confirm the six-factor model that was previously replicated in other studies, and also 2) examine its reliability, and convergent and divergent validity.

\section{Materials and Methods}

\section{Participants}

In total, 362 participants were included in the study (Table 1). They were all university students, their mean age was $21.12(\mathrm{SD}=3.07)$ years (range from 19 to 40 ), while $97.4 \%$ were younger than 30 years old. Age was not available from 48 participants, while gender was not reported by 58 participants. The majority of participants were female (57.7\%). Participation in the study was on a voluntary basis, no money or course credits were given to participants, and informed consent was obtained prior to the completion of research scales. The research was approved by the Ethics Committee of our University. Participants were all from the department of Educational and Social Policy of the University of Macedonia and were recruited from university classes.

Table 1. Demographics for study participants.

\begin{tabular}{cccc}
\hline & $\mathrm{N}$ & Mean age $(\mathrm{SD})$ & Age range \\
\hline Male & 118 & $21.0(3.4)$ & $19-44$ \\
Female & 178 & $21.3(2.9)$ & $19-43$ \\
Total & 296 & $21.1(3.1)$ & $19-44$ \\
\hline
\end{tabular}




\section{Procedure and Data collection}

The present study had correlational quantitative design. Firstly, OCI-R was translated from English to Greek and back to English by two authors. Finally, any differences were discussed until they reached a consensus. The rest of the scales were chosen after an extensive literature review to measure the convergent and divergent validity of OCI-R. The researchers recruited individuals from university classes that were interested in participating in the study. Participants were informed about the aim of the study and the procedure. Written informed consent was obtained from all participants. Individuals who agreed to participate in the study were given to complete a hard copy booklet which included a form with demographic information (gender, age) and all four questionnaires; the time to complete all questionnaires was approximately 20 minutes. These data were later digitalised by the researchers. Data analysis was done by IBM SPSS 21 for correlations and descriptive statistics and Lisrel for the Confirmatory Factor Analysis.

\section{Measures}

Obsessive-Compulsive Inventory-Revised (OCI-R; Foa et al., 2002). OCI-R measures distress caused by OCD symptoms. It consists of 18 items that are divided into six sub-scales: Washing, Checking, Ordering, Hoarding, Neutralization, and Obsessing. Participants' scores are rated in a 5-point scale $(0=$ Not at all, $1=$ A little, $2=$ Moderately, $3=\mathrm{A}$ lot, $4=$ Extremely). In the current study we used a Greek version of the OCI-R. Since the original OCI-R was copyrighted by Edna Foa (Foa et al., 2002) the first author (G.S) explicitly asked Foa's permission to have OCI-R translated into Greek, and Foa granted this permission. Consequently, the scale was translated from English to Greek and back to English by two authors (G.S \& O.Z.). Finally, any differences were discussed until the authors could reach a consensus and the accuracy of the translations was further reviewed. The internal consistency of the overall OCI-R in the present study proved to be excellent with Cronbach's Alpha $=.88$.

The Padua Inventory (PI; Sanavio, 1988) is a self-report measure of obsessive and compulsive symptoms, and it consists of 60 items describing common obsessional and compulsive behaviours. Four factors were identified: impaired control of mental activities (Cronbach's Alpha $=.93$ ), becoming contaminated (Cronbach's Alpha $=.92)$, checking behaviours (Cronbach's Alpha $=.92)$, urges and worries of losing control over motor behaviours (Cronbach's Alpha $=.89$ ). The PI is widely used in research on obsessions and compulsions and is available in various languages, as well as in Greek (Simos, 1997). The PI has acceptable reliability and validity in US, Italian, and Greek samples (Taylor, 1998; Sanavio, 1988; Simos, 1997; Sica, Taylor, Arrindell, \& Sanavio, 2006). In the present study, PI showed excellent internal consistency with Cronbach's Alpha $=.97$.

Obsessive Beliefs Questionnaire (OBQ-44; Obsessive Compulsive Cognitions Working Group, 2005) assesses belief domains related to OCD. It consists of 44 items that are divided into three factors: 1$)$ Responsibility and threat estimation. (Cronbach's Alpha $=.80), 2$ ) Perfectionism and intolerance for uncer- 
tainty (Cronbach's Alpha $=.80$ ), and 3) Importance and control of thoughts (Cronbach's Alpha $=.80$ ). In the present study OBQ-44 showed excellent internal consistency with Cronbach's Alpha $=.90$.

Depression Anxiety Stress Scale-21 (DASS-21; Lovibond \& Lovibond, 1995) describes negative feelings and the degree that the individual experiences these feelings. It consists of 21 items grouped into three subscales: Depression (Cronbach's Alpha $=.88)$, Anxiety (Cronbach's Alpha $=.79$ ), and Stress (Cronbach's Alpha $=.78)$. In the present study, we used the Greek version of DASS-21 (Lyrakos, Arvaniti, Smyrnioti, \& Kostopanagiotou, 2011). Lyrakos et al. (2011) translated DASS-21 into Greek using the multiple forward and backward translation protocol and checked the psychometric properties of the scale. The results of the original validation study suggest that the Greek translation of the DASS-21 is both reliable and valid, with psychometric properties close to those reported in the international literature. In the present study DASS-21 showed excellent internal consistency with Cronbach's Alpha $=.91$.

\section{Results}

\section{Factor Analysis}

Using Lisrel software we conducted a confirmatory factor analysis to test the goodness-of-fit of the hypothesized latent structure of the OCI-R (i.e., six correlated factors, each comprised of three items) (Figure 1). The residuals of the three items loading on each factor were correlated in the model. We estimated model fit via five indices: 1) chi-square, $\left.\mathrm{x}^{2}(120)=387.38, p<.001,2\right)$ an adjusted goodness of fit index of $.86,3$ ) a root mean square error of approximation of $.08,4)$ a comparative fit index of $.95,5$ ) and a normed fit index of .92 . These results replicate those reported by Foa et al. (2002) and indicate an adequate fit for the six-factor model of the OCI-R.

\section{Internal Consistency}

Table 2 shows mean scores, standard deviations, and internal consistency coefficients (Cronbach's Alpha) for OCI-R total score and subscales. Results showed good internal consistency with Alpha coefficients ranging from .64 to .80 .

The correlations between OCI-R total score and subscale scores were large, .69 (Neutralizing) to .74 (Checking). This suggested that the subscales all measured a common construct, OCD symptoms. The correlation between OCI-R subscale score were medium and ranged from $\mathrm{r}=.33$ ( $p<.000$; Washing-Hoarding) to $\mathrm{r}$ $=.49(p<.000$; Checking-Washing) suggesting that the symptom subscales were related but not redundant.

\section{Convergent Validity}

Convergent validity of the OCI-R total scale was estimated by correlating the OCI-R with OBQ-44 and PI. Bivariate Pearson correlations among the subscales of the OCI-R and the OBQ-44 (Table 3) were generally moderate (ranged from .18 to .48). Correlation among the subscales of the OCI-R and the PI (Table 4) ranged from medium to high (coefficients ranged from .30 to .64). 
Scatter plots for all pairs indicated that a non-linear measure of association was appropriate.

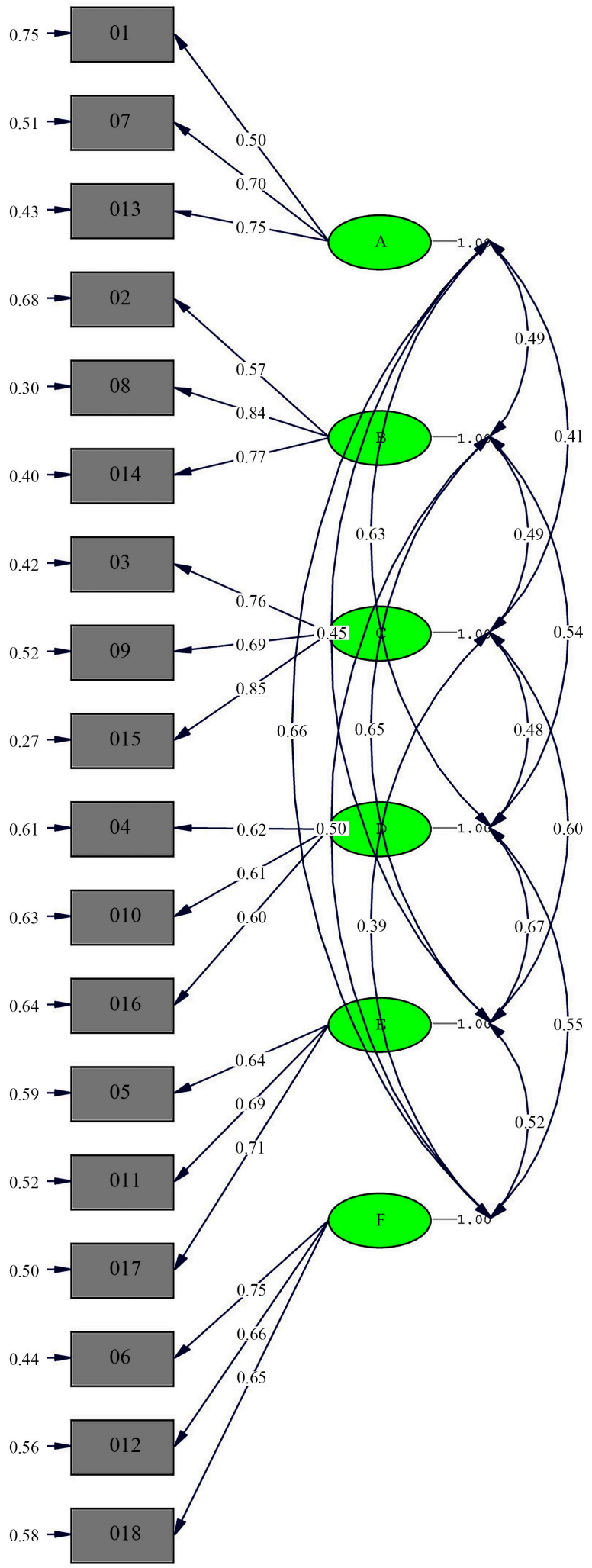

Figure 1. Confirmatory Factor analysis of OCI-R with Six factors $(n=362)$. 
Table 2. Internal Consistency (Cronbach's a), mean scores and standard deviations of OCI-R and its subscales $(n=362)$.

\begin{tabular}{cccc}
\hline OCI-R subscales & $\alpha$ & $M$ & Std. $D$. \\
\hline Checking & .76 & 3.7 & 3 \\
Hoarding & .67 & 3.6 & 2.8 \\
Neutralizing & .64 & 1.6 & 2.3 \\
Obsessing & .71 & 3.6 & 2.7 \\
Ordering & .80 & 4.8 & 3.2 \\
Washing & .71 & 2.8 & 2.7 \\
Total & .88 & 20.2 & 12 \\
\hline
\end{tabular}

Table 3. Bivariate Correlations between OCI-R and OBQ-44 total and subscales $(\mathrm{n}=$ 362).

\begin{tabular}{|c|c|c|c|c|}
\hline \multirow[b]{2}{*}{ OCI } & \multicolumn{4}{|c|}{ OBQ-44 } \\
\hline & $\begin{array}{l}\text { Responsibility/ } \\
\text { threat }\end{array}$ & $\begin{array}{l}\text { Perfectionism/ } \\
\text { certainty }\end{array}$ & $\begin{array}{c}\text { Importance control } \\
\text { thought }\end{array}$ & Total \\
\hline Checking & $.42^{\star \star}$ & $.33^{* *}$ & $.28^{\star *}$ & $.42^{\star *}$ \\
\hline Hoarding & $.34^{\star *}$ & $.20^{* *}$ & $.20^{\star *}$ & $.30^{* *}$ \\
\hline Neutralizing & $.35^{\star *}$ & $.31^{\star *}$ & $.29^{\star *}$ & $.38^{\star *}$ \\
\hline Obsessing & $.44^{\star *}$ & $.28^{\star *}$ & $.35^{\star \star}$ & $.44^{\star *}$ \\
\hline Ordering & $.30^{\star *}$ & $.28^{\star *}$ & $.21^{\star \star}$ & $.32^{\star *}$ \\
\hline Washing & $.31^{\star *}$ & $.30^{\star *}$ & $.25^{\star *}$ & $.36^{* *}$ \\
\hline Total & $.48^{\star *}$ & $.38^{* *}$ & $.34^{* *}$ & $.49^{\star *}$ \\
\hline
\end{tabular}

** correlation is significant at the 0.01 level (2-tailed).

Table 4. Bivariate Correlations between the OCI-R total score and subscales and PI total score and subscales $(\mathrm{n}=360)$.

\begin{tabular}{cccccc}
\hline \multicolumn{5}{c}{ Padua Inventory } \\
\hline OCI-R & Impaired control Contamination & Checking & Urges/ Worries & Total \\
\hline Checking & $.37^{* *}$ & $.29^{* *}$ & $.57^{* *}$ & .09 & $.39^{* *}$ \\
Hoarding & $.26^{* *}$ & $.13^{*}$ & $.17^{* *}$ & .02 & $.18^{* *}$ \\
Neutralizing & $.24^{* *}$ & $.15^{* *}$ & $.20^{* *}$ & .08 & $.22^{* *}$ \\
Obsessing & $.45^{* *}$ & $.23^{* *}$ & $.28^{* *}$ & $.15^{* *}$ & $.33^{* *}$ \\
Ordering & $.26^{* *}$ & $.29^{* *}$ & $.27^{* *}$ & .04 & $.28^{* *}$ \\
Washing & $.47^{* *}$ & $.63^{* *}$ & $.48^{* *}$ & $.25^{* *}$ & $.55^{* *}$ \\
Total & $.47^{* *}$ & $.41^{* *}$ & $.47^{* *}$ & $.14^{* *}$ & $.46^{* *}$ \\
\hline
\end{tabular}

${ }^{*}$ correlation is significant at the 0.05 level (2-tailed), ${ }^{* *}$ correlation is significant at the 0.01 level (2-tailed). 


\section{Divergent Validity}

Divergent validity of OCI-R was assessed by correlating the OCI-R total and subscale scores with DASS-21, a measure of depression, anxiety and tension/stress (Table 5). Concerning correlation among OCI-R subscales on the one hand, and DASS-21subscales on the other, most correlation coefficients (11 out of 18) were smaller than .30 , while the rest ranged from .30 to .46 .

Overall, the results of the present study supported the hypothesis that the Greek version of OCI-R would show good psychometric properties. The results showed acceptable good divergent and convergent validity. Results of the confirmatory factor analysis were in accordance with the ones of the original version and all other studies that focused on the psychometric properties of the OCI-R.

\section{Discussion}

The present study aimed to examine the psychometric properties of OCI-R in Greek language in a non-clinical sample. Present results showed that the Greek version of OCI-R maintained the psychometric properties of the original version introduced by Foa et al. (2002).

In accordance with the original version of the OCI-R, the factor structure of the scale produced six independent factors: Checking, Ordering, Obsessing, Hoarding, Washing and Neutralizing. The confirmatory factor analysis showed good fit of the model and replicated the results of other studies meeting the requirements for the six-factor solution of the original scale (Gönner et al., 2008; Fullana et al., 2005; Zermatten et al., 2006; Woo et al., 2010; Foa et al., 2002).

The internal consistency was excellent for the overall scale and very good to excellent for four of the sub-scales: Checking, Obsessing, Ordering and Washing. The Hoarding and Neutralizing sub-scales showed an internal consistency lower than the recommended value (Alpha $<.70$ ). These results are similar to the ones of the original and other consequent studies that adapted OCI-R in different cultural settings or populations (e.g., Foa et al., 2002; Smári et al., 2007; Gönner et al., 2008). An explanation for the low internal consistency coefficient of the Neutralizing subscale may be that Neutralizing is rare in non-OCD individuals and the low Alpha coefficient may reflect a restricted range rather than a structural inadequacy (Sica et al., 2009). Additionally, other researchers have suggested a revision of the Neutralizing scale because the content of the items may fail to capture many clinically relevant neutralization phenomena (Gönner et al., 2008; Purdon \& Clark, 2002).

Convergent validity was examined by calculating the correlation between the OCI-R and two measures of OCD constructs, OBQ-44 and PI. Results showed moderate correlations, confirming that OCI-R has been developed to measure symptoms that indicate OCD. Similar findings have been reported by Zermatten et al. (2006), Sica et al. (2009) and Fullana et al. (2005), although in their studies correlations between OCI-R and PI were higher than the ones in the present study. These differences may be explained by variations in samples used across studies (age, sampling methods, cultural differences etc.). 
Table 5. Bivariate Correlations between OCI-R and DASS-21 total and subscales $(\mathrm{n}=$ 362).

\begin{tabular}{ccccc}
\hline & \multicolumn{3}{c}{ DASS } \\
\hline OCI-R & Depression & Anxiety & Stress & Total \\
\hline Checking & $.201^{* *}$ & $.289^{* *}$ & $.251^{* *}$ & $.280^{* *}$ \\
Hoarding & $.344^{* *}$ & $.270^{* *}$ & $.366^{* *}$ & $.379^{* *}$ \\
Neutralizing & $.209^{* *}$ & $.311^{* *}$ & $.222^{* *}$ & $.278^{* *}$ \\
Obsessing & $.456^{* *}$ & $.404^{* *}$ & $.459^{* *}$ & $.507^{* *}$ \\
Ordering & $.197^{* *}$ & $.260^{* *}$ & $.299^{* *}$ & $.288^{* *}$ \\
Washing & $.221^{* *}$ & $.253^{* *}$ & $.205^{* *}$ & $.257^{* *}$ \\
Total & $.378^{* *}$ & $.415^{* *}$ & $.423^{* *}$ & $.464^{* *}$ \\
\hline
\end{tabular}

${ }^{* *}$ Correlation is significant at the 0.01 level (2-tailed).

All subscales of the OCI-R correlated significantly with low to moderate correlations with OBQ-44, confirming the well-known relationship between OCD symptoms and relevant belief domains (Obsessive Compulsive Cognitions Working Group, 2001). Among OCI-R subscales, the subscale of Checking had one of the highest correlation coefficients with the OBQ-44 subscale of Responsibility/threat. Tolin, Woods, \& Abramowitz (2003) in their study with a similar student sample also found that the highest correlation of OCI-R Checking was associated with the Threat estimation belief domain of OBQ-44. Another finding in the present study was that the subscale of Obsessing also had one of the highest correlations to Responsibility/threat estimation and Importance/control of thoughts, a finding that is in agreement with the prominent role of these two domains in cognitive theories of OCD (Salkovskis, Shafran, Rachman, \& Freeston, 1999; Thordarson \& Shafran, 2002), and were also on partial agreement with the Tolin et al. study in a clinical sample (Tolin, Brady, \& Hannan, 2008) where OCI-R Obsessing was predicted by Importance/control of thoughts, but not Responsibility/threat. Interestingly, we found a stronger correlation of Obsessing with the domain of Responsibility/threat than the domain of Importance/control of thoughts, something that traditionally is the other way round (Tolin et al., 2008).

Our finding of the lowest correlation between the Hoarding subscale and Perfectionism/uncertainty, Importance/control of thoughts, and OBQ-44 total score, which has also been reported in other studies (e.g., Foa et al., 2002; Woo et al., 2010), may also suggest that hoarding may not be a fully representative feature of OCD (Grisham \& Norberg, 2010). These data are in congruence to the clinical practice, as the Fifth Edition of the Diagnostic and Statistical Manual of Mental Disorders-DSM-5 (APA, 2013) considers hoarding as a distinct disorder from OCD, although it also acknowledges a significant relationship between the two, including thus both Hoarding Disorder and OCD in the new category of Obsessive-Compulsive and Related Disorders. This change will likely have a 
considerable impact on the self-report scales that assess symptoms of OCD, as these scales often include items measuring symptoms of hoarding.

Furthermore, in the correlations between OCI-R and PI, the OCI-R Washing subscale had the highest correlations with the majority of PI subscales, except from the PI subscale of Urges/worries, which generally had no significant correlations with the OCI-R scale. The OCI-R Washing subscale demonstrated high correlations with the PI subscales of Contamination $(r=.63)$, the OCI-R Checking subscale showed high correlation with the PI Checking subscale $(r=.57)$, and the OCI-I subscale of Obsessing showed high correlation with the PI subscale of Impaired Control $(r=.45)$. Such findings are also conceptually significant, and also in congruence with previous studies that also found good correlations among these subscales (Fullana et al., 2005; Sica et al., 2009).

Divergent Validity was examined by calculating the correlation between the OCI-R and DASS-21. Results showed low correlation of the OCI-R with the Depression subscale, and moderate correlation with the subscales of Anxiety and Stress. That is in partial agreement with the idea that, although in practice obsessive-compulsive symptoms usually seem to coexist with depressive and anxiety symptoms, correlations of the total OCI-R with measures of anxiety, worry, and depression are low to moderate (Gönner et al., 2008). Therefore we can interpret our findings by considering the undisputable high comorbidity of obsessive-compulsive symptoms with anxiety (Gönner et al., 2008). Furthermore, the low correlation of OCI-R with the DASS-21 Depression subscale supports an adequate degree of divergent validity.

The vast majority of the studies that have validated OCI-R in other cultural settings or re-examined OCI-R's psychometric properties, have reported the existence of a good or adequate discriminant validity (Hajcak et al., 2004; Fullana et al., 2005; Sica et al., 2009; Smári et al., 2007; Woo et al., 2010). However, in our study correlations were higher than expected according to previous research findings. Among the OCI-R subscales, the Obsessing one had the highest correlation with Depression, Anxiety, and Stress, and such relationships may thus have resulted in the elevation of the DASS-21 total score. An explanation for this finding may be in the influence of a mediating factor that may be common among obsessing, depression and anxiety. Obsessing usually involves rumination which is also found in other disorders, such as depression and anxiety (McLaughlin \& Nolen-Hoeksema, 2011; Nolen-Hoeksema, 2000). One can guess that there is no much difference between item \#6 (Obsessing) of OCI-R "I find it difficult to control my own thoughts" and item \#7 of the Perseverative Thinking Questionnaire (PTQ; Ehring, Zetsche, Weidacker, Wahl, Schönfeld et al., 2011) “Thoughts come to my mind without me wanting them to". Ehring et al. (2011) developed PTQ as a transdiagnostic process measure of repetitive negative thinking and found that it significantly correlates with symptom levels of both depression and anxiety.

Several key limitations should be noted. Firstly, participants were all university students, and therefore, the results might not necessarily be representative of 
the general Greek population. Secondly, the sample was rather small and does not also allow for generalisation. Finally, the study was conducted in a non-clinical sample; relationships among the variables we examined may be somewhat different in an appropriate clinical sample. Nevertheless, and despite the above limitations, results in the present study support the adequate psychometric properties of the Greek version of OCI-R. Taking into consideration the limited research data from the application of OCI-R in Greek clinical samples, one expects that future studies will focus on this field.

\section{Conclusion}

To conclude, the Greek version of OCI-R is a sound psychometric tool with good psychometric properties in a non-clinical young adult student population. Results of the present study add to the body of studies in different languages and cultures. Similar findings were also reported by Angelakis et al. (2017), who also confirmed the psychometric properties of OCI-R in a Greek community sample. Taking into consideration the Angelakis et al. (2017) study and our study, it is interesting that although our sample was a student only sample, and we used different questionnaires to test convergent and divergent validities of OCI-R, we both arrived at similar results and conclusions. As a closing remark, one has also to consider that there is still an ongoing need for validated measures for the assessment of OCD symptoms and related constructs in Greece.

\section{Conflicts of Interest}

The authors declare no conflicts of interest regarding the publication of this paper.

\section{References}

Abramowitz, J. S., Deacon, B. J., Olatunji, B. O., Wheaton, M. G., Berman, N. C., Losardo, D., Björgvinsson, T. et al. (2010). Assessment of Obsessive-Compulsive Symptom Dimensions: Development and Evaluation of the Dimensional Obsessive-Compulsive Scale. Psychological Assessment, 22, 180-198. https://doi.org/10.1037/a0018260

Abramowitz, J. S., Tolin, D. F., \& Diefenbach, G. J. (2005). Measuring Change in OCD: Sensitivity of the Obsessive-Compulsive Inventory-Revised. Journal of Psychopathology and Behavioral Assessment, 27, 317-324. https://doi.org/10.1007/s10862-005-2411-y

Abramowitz, J., \& Deacon, B. (2006). Psychometric Properties and Construct Validity of the Obsessive-Compulsive Inventory-Revised: Replication and Extension with a Clinical Sample. Journal of Anxiety Disorders, 20, 1016-1035.

https://doi.org/10.1016/j.janxdis.2006.03.001

Altamura, A. C., Buoli, M., Albano, A., \& Dell'Osso, B. (2010). Age at Onset and Latency to Treatment (Duration of Untreated Illness) in Patients with Mood and Anxiety Disorders: A Naturalistic Study. International Clinical Psychopharmacology, 25, 172-179. https://doi.org/10.1097/YIC.0b013e3283384c74

American Psychiatric Association (APA) (2013). Diagnostic and Statistical Manual of Mental Disorders (DSM-5 ${ }^{\circledR}$ ). Washington DC: American Psychiatric Publishing. https://doi.org/10.1176/appi.books.9780890425596 
Angelakis, I., Panagioti, M., \& Austin, J. (2017). Factor Structure and Validation of the Obsessive-Compulsive Inventory-Revised (OCI-R) in a Greek Non-Clinical Sample. Journal of Psychopathology and Behavioral Assessment, 39, 164-175. https://doi.org/10.1007/s10862-016-9575-5

Arafat, S. M., Chowdhury, H. R., Qusar, M. S., \& Hafez, M. A. (2016). Cross Cultural Adaptation \& Psychometric Validation of Research Instruments: A Methodological Review. Journal of Behavioral Health, 5, 129-136. https://doi.org/10.5455/jbh.20160615121755

Aydin, A., Boysan, M., Kalafat, T., Selvi, Y., Beşiroğlu, L., \& Kagan, M. (2014). Validation of the Turkish Version of the Obsessive-Compulsive Inventory-Revised (OCI-R) in Clinical and Non-Clinical Samples. Nöropsikiyatri Arşivi, 51, 15. https://doi.org/10.1037/t63941-000

Beck, A. T., Rush, A. J., Shaw, B. F., \& Emery, G. (1979). Cognitive Therapy of Depression. New York: Guilford.

Belloch, A., del Valle, G., Morillo, C., Carrió, C., \& Cabedo, E. (2008). To Seek Advice or Not to Seek Advice about the Problem: The Help-Seeking Dilemma for Obsessive-Compulsive Disorder. Social Psychiatry and Psychiatric Epidemiology, 44, 257-264. https://doi.org/10.1007/s00127-008-0423-0

Belloch, A., Roncero, M., García-Soriano, G., Carrió, C., Cabedo, E., \& Fernández-Álvarez, H. (2013). The Spanish Version of the Obsessive-Compulsive Inventory-Revised (OCI-R): Reliability, Validity, Diagnostic Accuracy, and Sensitivity to Treatment Effects in Clinical Samples. Journal of Obsessive-Compulsive and Related Disorders, 2, 249-256. https://doi.org/10.1016/j.jocrd.2013.05.001

Bloch, M., Landeros-Weisenberger, A., Rosario, M., Pittenger, C., \& Leckman, J. (2008). Meta-Analysis of the Symptom Structure of Obsessive-Compulsive Disorder. American Journal of Psychiatry, 165, 1532-1542. https://doi.org/10.1176/appi.ajp.2008.08020320

Burns, G. L., Keortge, S. G., Formea, G. M., \& Sternberger, L. G. (1996). Revision of the Padua Inventory of Obsessive-Compulsive Disorder Symptoms: Distinctions between Worry, Obsessions, and Compulsions. Behaviour Research and Therapy, 34, 163-173. https://doi.org/10.1016/0005-7967(95)00035-6

Clark, D. A. (2004). Cognitive-Behavioral Therapy for OCD. New York: Guilford Press.

Clark, D. A., Antony, M. M., Beck, A. T., Swinson, R. P., \& Steer, R. A. (2005). Screening for Obsessive and Compulsive Symptoms: Validation of the Clark-Beck Obsessive-Compulsive Inventory. Psychological Assessment, 17, 132. https://doi.org/10.1037/1040-3590.17.2.132

Ehring, T., Zetsche, U., Weidacker, K., Wahl, K., Schönfeld, S., \& Ehlers, A. (2011). The Perseverative Thinking Questionnaire (PTQ): Validation of a Content-Independent Measure of Repetitive Negative Thinking. Journal of Behavior Therapy and Experimental Psychiatry, 42, 225-232. https://doi.org/10.1016/j.jbtep.2010.12.003

Ercikan, K. (2002). Disentangling Sources of Differential Item Functioning in Multilanguage Assessments. International Journal of Testing, 2, 199-215. https://doi.org/10.1207/S15327574IJT023\&4_2

Foa, E. B., Huppert, J. D., Leiberg, S., Langner, R., Kichic, R., Hajcak, G., \& Salkovskis, P. M. (2002). The Obsessive-Compulsive Inventory: Development and Validation of a Short Version. Psychological Assessment, 14, 485. https://doi.org/10.1037/1040-3590.14.4.485

Foa, E., Kozak, M., Salkovskis, P., Coles, M., \& Amir, N. (1998). The Validation of a New Obsessive-Compulsive Disorder Scale: The Obsessive-Compulsive Inventory. Psychological Assessment, 10, 206-214. https://doi.org/10.1037/1040-3590.10.3.206 
Fullana, M., Tortella-Feliu, M., Caseras, X., Andión, Ó., Torrubia, R., \& Mataix-Cols, D. (2005). Psychometric Properties of the Spanish Version of the Obsessive-Compulsive Inventory-Revised in a Non-Clinical Sample. Journal of Anxiety Disorders, 19, 893-903. https://doi.org/10.1016/j.janxdis.2004.10.004

García-Soriano, G., Rufer, M., Delsignore, A., \& Weidt, S. (2014). Factors Associated with Non-Treatment or Delayed Treatment Seeking in OCD Sufferers: A Review of the Literature. Psychiatry Research, 220, 1-10. https://doi.org/10.1016/j.psychres.2014.07.009

Ghassemzadeh, H., Shams, G., Abedi, J., Karamghadiri, N., Ebrahimkhani, N., \& Rajabloo, M. (2011). Psychometric Properties of a Persian-Language Version of the Obsessive-Compulsive Inventory-Revised: OCI-R-Persian. Psychology, 2, 210-215. https://doi.org/10.4236/psych.2011.23032

Gönner, S., Ecker, W., \& Leonhart, R. (2009). Diagnostic Discrimination of Patients with Different OCD Main Symptom Domains from Each Other and from Anxious and Depressive Controls. Journal of Psychopathology and Behavioral Assessment, 31, 159-167. https://doi.org/10.1007/s10862-008-9114-0

Gönner, S., Leonhart, R., \& Ecker, W. (2008). The Obsessive-Compulsive Inventory-Revised (OCI-R): Validation of the German Version in a Sample of Patients with OCD, Anxiety Disorders, and Depressive Disorders. Journal of Anxiety Disorders, 22, 734-749. https://doi.org/10.1016/j.janxdis.2007.07.007

Goodman, W. K., Price, L. H., Rasmussen, S. A., Mazure, C., Fleischmann, R. L., Hill, C. L., Charney, D. S. et al. (1989). Yale-Brown Obsessive Compulsive Scale (Y-BOCS). Archives of General Psychiatry, 46, 1006-1011. https://doi.org/10.1001/archpsyc.1989.01810110048007

Grisham, J. R., \& Norberg, M. M. (2010). Compulsive Hoarding: Current Controversies and New Directions. Dialogues in Clinical Neuroscience, 12, 233.

Hajcak, G., Huppert, J., Simons, R., \& Foa, E. (2004). Psychometric Properties of the OCI-R in a College Sample. Behaviour Research and Therapy, 42, 115-123. https://doi.org/10.1016/j.brat.2003.08.002

Hambleton, R. K., Merenda, P. F., \& Spielberger, C. D. (2004). Issues, Designs, and Technical Guidelines for Adapting Tests into Multiple Languages and Cultures. In Adapting Educational and Psychological Tests for Cross-Cultural Assessment (pp. 15-50). New York: Psychology Press. https://doi.org/10.4324/9781410611758

Hodgson, R. J., \& Rachman, S. (1977). Obsessional-Compulsive Complaints. Behaviour Research and Therapy, 15, 389-395. https://doi.org/10.1016/0005-7967(77)90042-0

Huppert, J., Walther, M., Hajcak, G., Yadin, E., Foa, E., Simpson, H., \& Liebowitz, M. (2007). The OCI-R: Validation of the Subscales in a Clinical Sample. Journal of Anxiety Disorders, 21, 394-406. https://doi.org/10.1016/j.janxdis.2006.05.006

Lovibond, P. F., \& Lovibond, S. H. (1995). The Structure of Negative Emotional States: Comparison of the Depression Anxiety Stress Scales (DASS) with the Beck Depression and Anxiety Inventories. Behaviour Research and Therapy, 33, 335-343. https://doi.org/10.1016/0005-7967(94)00075-U

Lyrakos, G. N., Arvaniti, C., Smyrnioti, M., \& Kostopanagiotou, G. (2011). P03-561Translation and Validation Study of the Depression Anxiety Stress Scale in the Greek General Population and in a Psychiatric Patient's Sample. European Psychiatry, 26, 1731. https://doi.org/10.1016/S0924-9338(11)73435-6

McKay, D., Abramowitz, J., Calamari, J., Kyrios, M., Radomsky, A., Sookman, D. et al. (2004). A Critical Evaluation of Obsessive-Compulsive Disorder Subtypes: Symptoms versus Mechanisms. Clinical Psychology Review, 24, 283-313.

https://doi.org/10.1016/j.cpr.2004.04.003 
McLaughlin, K., \& Nolen-Hoeksema, S. (2011). Rumination as a Transdiagnostic Factor in Depression and Anxiety. Behaviour Research and Therapy, 49, 186-193. https://doi.org/10.1016/j.brat.2010.12.006

Meyer, T. J., Miller, M. L., Metzger, R. L., \& Borkovec, T. D. (1990). Development and Validation of the Penn State Worry Questionnaire. Behaviour Research and Therapy, 28, 487-495. https://doi.org/10.1016/0005-7967(90)90135-6

Nolen-Hoeksema, S. (2000). The Role of Rumination in Depressive Disorders and Mixed Anxiety/Depressive Symptoms. Journal of Abnormal Psychology, 109, 504-511. https://doi.org/10.1037/0021-843X.109.3.504

Obsessive Compulsive Cognitions Working Group (2001). Development and Initial Validation of the Obsessive Beliefs Questionnaire and the Interpretation of Intrusions Inventory. Behaviour Research and Therapy, 39, 987-1006. https://doi.org/10.1016/S0005-7967(00)00085-1

Obsessive Compulsive Cognitions Working Group (2005). Psychometric Validation of the Obsessive Belief Questionnaire and Interpretation of Intrusions Inventory-Part 2: Factor Analyses and Testing of a Brief Version. Behaviour Research and Therapy, 43, 1527-1542. https://doi.org/10.1016/j.brat.2004.07.010

Olatunji, B. O., Williams, B. J., Haslam, N., Abramowitz, J. S., \& Tolin, D. F. (2008). The Latent Structure of Obsessive-Compulsive Symptoms: A Taxometric Study. Depression and Anxiety, 25, 956-968. https://doi.org/10.1002/da.20387

Peng, Z., Yang, W., Miao, G., Jing, J., \& Chan, R. (2011). The Chinese Version of the Obsessive-Compulsive Inventory-Revised Scale: Replication and Extension to Non-Clinical and Clinical Individuals with OCD Symptoms. BMC Psychiatry, 11, 129.

https://doi.org/10.1186/1471-244X-11-129

Politis, S., Magklara, K., Petrikis, P., Michalis, G., Simos, G., \& Skapinakis, P. (2017). Epidemiology and Comorbidity of Obsessive-Compulsive Disorder in Late Adolescence: A Cross-Sectional Study in Senior High Schools in Greece. International Journal of Psychiatry in Clinical Practice, 21, 188-194. https://doi.org/10.1080/13651501.2017.1324038

Purdon, C., \& Clark, D. A. (2002). The Need to Control Thoughts. In Cognitive Approaches to Obsessions and Compulsions (pp. 29-43). London: Pergamon. https://doi.org/10.1016/B978-008043410-0/50004-0

Rapp, A. M., Bergman, R. L., Piacentini, J., \& McGuire, J. F. (2016). Evidence-Based Assessment of Obsessive-Compulsive Disorder. Journal of Central Nervous System Disease, 8 , JCNSD-S38359. https://doi.org/10.4137/JCNSD.S38359

Salkovskis, P., Shafran, R., Rachman, S., \& Freeston, M. H. (1999). Multiple Pathways to Inflated Responsibility Beliefs in Obsessional Problems: Possible Origins and Implications for Therapy and Research. Behaviour Research and Therapy, 37, 1055-1072. https://doi.org/10.1016/S0005-7967(99)00063-7

Sanavio, E. (1988). Obsessions and Compulsions: The Padua Inventory. Behaviour Research and Therapy, 26, 169-177. https://doi.org/10.1016/0005-7967(88)90116-7

Sica, C., Ghisi, M., Altoè, G., Chiri, L., Franceschini, S., Coradeschi, D., \& Melli, G. (2009). The Italian Version of the Obsessive-Compulsive Inventory: Its Psychometric Properties on Community and Clinical Samples. Journal of Anxiety Disorders, 23, 204-211. https://doi.org/10.1016/j.janxdis.2008.07.001

Sica, C., Taylor, S., Arrindell, W. A., \& Sanavio, E. (2006). A Cross-Cultural Test of the Cognitive Theory of Obsessions and Compulsions: A Comparison of Greek, Italian, and American Individuals-A Preliminary Study. Cognitive Therapy and Research, 30, 585-597. https://doi.org/10.1007/s10608-006-9078-x 
Simonds, L. M., Thorpe, S. J., \& Elliott, S. A. (2000). The Obsessive-Compulsive Inventory: Psychometric Properties in a Nonclinical Student Sample. Behavioural and Cognitive Psychotherapy, 28, 153-159. https://doi.org/10.1017/S1352465800001065

Simos, G. (1997). The Greek Version of the Padua Inventory. Unpublished Manuscript, Thessaloniki, Greece: Department of Psychiatry, Central District 2nd University.

Simos, G., \& Alexandri, E. (2014). Obsessive Compulsive Inventory-Revised: Application to and Its Psychometric Characteristics in a Sample of Greek Young Adults. Psychiatriki, 25.

Skapinakis, P., Bellos, S., Koupidis, S., Grammatikopoulos, I., Theodorakis, P. N., \& Mavreas, V. (2013). Prevalence and Sociodemographic Associations of Common Mental Disorders in a Nationally Representative Sample of the General Population of Greece. BMC Psychiatry, 13, 163. https://doi.org/10.1186/1471-244X-13-163

Smári, J., Ólason, D., EyPórsdóttir, Á., \& Frölunde, M. (2007). Psychometric Properties of the Obsessive-Compulsive Inventory-Revised among Icelandic College Students. Scandinavian Journal of Psychology, 48, 127-133. https://doi.org/10.1111/j.1467-9450.2007.00574.x

Sousa, V. D., \& Rojjanasrirat, W. (2011). Translation, Adaptation and Validation of Instruments or Scales for Use in Cross-Cultural Health Care Research: A Clear and User-Friendly Guideline. Journal of Evaluation in Clinical Practice, 17, 268-274. https://doi.org/10.1111/j.1365-2753.2010.01434.x

Souza, F., Foa, E., Meyer, E., Niederauer, K., \& Cordioli, A. (2011). Psychometric Properties of the Brazilian Portuguese Version of the Obsessive-Compulsive Inventory: Revised (OCI-R). Revista Brasileira De Psiquiatria, 33, 137-142. https://doi.org/10.1590/S1516-44462011000200008

Taylor, S. (1998). Assessment of Obsessive-Compulsive Disorder. In R. P. Swinson, M. M. Antony, S. Rachman, \& M. A. Richter (Eds.), Obsessive-Compulsive Disorder: Theory, Research, and Treatment (pp. 229-257). New York: Guilford.

Thordarson, D. S., \& Shafran, R. (2002). Importance of Thoughts. In Cognitive Approaches to Obsessions and Compulsions (pp. 15-28). London: Pergamon. https://doi.org/10.1016/B978-008043410-0/50003-9

Thordarson, D. S., Radomsky, A. S., Rachman, S., Shafran, R., Sawchuk, C. N., \& Hakstian, A. R. (2004). The Vancouver Obsessional Compulsive Inventory (VOCI). Behaviour Research and Therapy, 42, 1289-1314. https://doi.org/10.1016/j.brat.2003.08.007

Tolin, D. F., Brady, R. E., \& Hannan, S. (2008). Obsessional Beliefs and Symptoms of Obsessive-Compulsive Disorder in a Clinical Sample. Journal of Psychopathology and Behavioral Assessment, 30, 31-42. https://doi.org/10.1007/s10862-007-9076-7

Tolin, D. F., Woods, C. M., \& Abramowitz, J. S. (2003). Relationship between Obsessive Beliefs and Obsessive-Compulsive Symptoms. Cognitive Therapy and Research, 27, 657-669. https://doi.org/10.1023/A:1026351711837

Wahl, K., Kordon, A., Kuelz, K. A., Voderholzer, U., Hohagen, F., \& Zurowski, B. (2010). Obsessive-Compulsive Disorder (OCD) Is Still an Unrecognised Disorder: A Study on the Recognition of OCD in Psychiatric Outpatients. European Psychiatry, 25, 374-377. https://doi.org/10.1016/j.eurpsy.2009.12.003

Williams, M., Davis, D., Thibodeau, M., \& Bach, N. (2013). Psychometric Properties of the Obsessive-Compulsive Inventory Revised in African Americans with and without Obsessive-Compulsive Disorder. Journal of Obsessive-Compulsive and Related Disorders, 2, 399-405. https://doi.org/10.1016/j.jocrd.2013.07.003

Woo, C., Kwon, S., Lim, Y., \& Shin, M. (2010). The Obsessive-Compulsive Inventory-Revised (OCI-R): Psychometric Properties of the Korean Version and the Order, 
Gender, and Cultural Effects. Journal of Behavior Therapy and Experimental Psychiatry, 41, 220-227. https://doi.org/10.1016/j.jbtep.2010.01.006

Zermatten, A., Van der Linden, M., Jermann, F., \& Ceschi, G. (2006). Validation of a French Version of the Obsessive-Compulsive Inventory-Revised in a Non-Clinical Sample. Revue Européenne De Psychologie Appliquée/European Review of Applied Psychology, 56, 151-155. https://doi.org/10.1037/0021-843X.109.3.504 